\title{
The Aurora Kinase A Polymorphisms are not Associated with Recurrence- free Survival in Prostate Cancer Patients
}

\author{
Jerry J. Jaboin ${ }^{1}$, Natalie L. Ausborn ${ }^{1}$, Misun Hwang ${ }^{1}$, Heidi Chen ${ }^{2}$, Kenneth J. Niermann ${ }^{1}$, Nicholas J. Giacalone ${ }^{1}$, Regina Courtney ${ }^{3,4}$ \\ Qiuyin $\mathrm{Cai}^{3,4}$ and Bo Lu ${ }^{5 *}$
}

${ }^{1}$ Department of Radiation Oncology, Vanderbilt University School of Medicine, USA

${ }^{2}$ Department of Biostatistics, Vanderbilt University, USA

${ }^{3}$ Department of Medicine, Vanderbilt University School of Medicine, USA

${ }^{4}$ Vanderbilt Epidemiology Center, Vanderbilt University Medical Center, USA

${ }^{5}$ Department of Radiation Oncology, Jefferson Medical College of Thomas Jefferson University, USA

\begin{abstract}
Background: The purpose of this study was to investigate the association between haplotype-tagging single nucleotide polymorphisms (SNPs) within the Aurora Kinase A (AURKA) gene and prostate cancer outcomes in patients with clinically localized prostate cancer.

Methods: Four intronic haplotype-tagging SNPs within the AURKA gene were individually selected and examined in regard to their influence on clinical outcomes in 212 patients who underwent radical prostatectomy as first-line treatment. Haplotype-tagging SNPs were selected using the ABI SNP Browser to cover SNPs with an $\mathrm{r}^{2}$ of 0.90 or greater in the AURKA gene with a minor allele frequency of at least 0.25 .

Results: In our study, a log-rank univariate analysis was performed to identify significant associations between probability of recurrence-free survival or disease-free survival and known prognostic indicators as well as AURKA genotypes. The prognostic indicators Gleason grade, surgical margin, extracapsular extension, and disease stage were associated with recurrence-free survival (all $p<0.001)$. Only Gleason grade was associated with disease-free survival $(p<0.001)$. No associations were found for the SNPs $r s 1468055, r s 8117896, r s 2064863$, and $r s 1468056$ analyzed for either RFS $(p=0.7213, p=0.5140, p=0.0714, p=0.4731$, respectively) or DFS $(p=0.3282, p=0.1909$, $p=0.4111, p=0.5014$, respectively).
\end{abstract}

Conclusions: This study demonstrates no evidence for intronic AURKA SNPs in predicting recurrence-free survival in patients with prostate cancer.

Keywords: Aurora kinase A; Prostate cancer; Single nucleotide polymorphism; Recurrence-free survival

Abbreviations: AURKA: Aurora Kinase A; DFS: Disease-free Survival; ht-SNP: Haplotype-tagging Single Nucleotide Polymorphism; PSA: Prostate-specific Antigen; RFS: Recurrence-free Survival; SNP: Single Nucleotide Polymorphism

\section{Introduction}

Aurora kinase A (also known as STK15, BTAK, AIKI, and AURKA) is a well-known oncogene and serine/threonine kinase, which localizes to centrosomes in $\mathrm{S}$ phase to undergo phosphorylation and activation late in G2 phase. Human AURKA localizes to chromosome 20q13.2, a region known to be frequently amplified in multiple tumor types $[1,2]$. Its overexpression has been associated with centrosome amplification, chromosomal instability and malignant phenotypes of mammalian cells [3]. Overexpression of AURKA has been noted in prostate tumors in human and mouse models [4], and its link to tumor progression and metastasis have been demonstrated [5]. Several studies have shown that the overexpression of AURKA is more relevant in early tumorigenesis $[6,7]$, whereas others have emphasized its role in tumor progression [8].

Ongoing genetic studies on AURKA have revealed two functional AURKA SNPs, rs2273535 and rs1047972, that can affect AURKA promoter activity $[9,10]$ and promote tumor progression in multiple cancer types, including prostate cancer [9]. Heretofore, no followup epidemiologic studieshave been reported describing the potential association between these functional AURKA SNPs and clinical outcomes in prostate cancer. Additionally, to our knowledge, no AURKA SNPs have been reported in any prostate cancer genome wide association studies. Further studies are necessary given the prognostic utility of SNPs in predicting cancer risk and recurrence [11,12], based on the premise that these SNP studies will enhance the accuracy of existing prognostic nomograms that incorporate clinical variables such as clinical stage, Gleason score, and prostate-specific antigen (PSA) levels [13].

Interestingly, recent studies on apoptotic and cell cycle genes have revealed a pivotal role of introns in transcriptional regulation, suggesting the potential importance of intronic SNPs in studying cancer risk-stratification. In the study herein, we analyzed the four intronic AURKA SNPs to determine their prognostic outcome associations in clinically localized prostate cancer after radical prostatectomy.

*Corresponding author: Bo Lu, MD, PhD, Department of Radiation Oncology, Jefferson Medical College of Thomas Jefferson University, 111 South 11th Street Room G-301N, Bodine Center, Philadelphia, PA 19107, USA, Tel: 215-955-6705 Fax: 215-955-0412; E-mail: bo.lu@jeffersonhospital.org

Received December 08, 2011; Accepted February 07, 2012; Published February 09,2012

Citation: Jaboin JJ, Ausborn NL, Hwang M, Chen H, Niermann KJ, et al. (2012) The Aurora Kinase A Polymorphisms are not Associated with Recurrence-free Survival in Prostate Cancer Patients. J Cancer Sci Ther 4: 016-022. doi:10.4172/19485956.1000105

Copyright: @ 2012 Jaboin JJ, et al. This is an open-access article distributed unde the terms of the Creative Commons Attribution License, which permits unrestricted use, distribution, and reproduction in any medium, provided the original author and source are credited. 
Citation: Jaboin JJ, Ausborn NL, Hwang M, Chen H, Niermann KJ, et al. (2012) The Aurora Kinase A Polymorphisms are not Associated with Recurrence-free Survival in Prostate Cancer Patients. J Cancer Sci Ther 4: 016-022. doi:10.4172/1948-5956.1000105

\section{Methods}

\section{Study population and clinical data}

Patient population consisted of 212 predominantly Caucasian prostate cancer patients status-post radical prostatectomy at Vanderbilt University Hospital between 1997 and 1999. Patient consent and approval by the Vanderbilt University Institutional Review Board preceded the collection of prostate tissues for research. Histologic confirmation of all prostate samples revealed adenocarcinoma. Clinical data on patient follow-up at Vanderbilt University Medical Center were retrospectively gathered via electronic medical records. The mean follow-up for disease-free survival and assessment of prostate cancer progression were $8.3 \pm 2.4-\mathrm{y}$ and $4.4 \pm 3.9$-y, respectively. The endpoints analyzed were recurrence-free survival (RFS) and diseasefree survival (DFS). Recurrence post-prostatectomy was classified as biochemical (which has been shown to be a poor predictor of overall survival in patients with localized prostate cancer treated with radical prostatectomy) [14], local or distant, and the most advanced recurrence type documented was assigned to each patient. Biochemical recurrence was defined as a prostate-specific antigen (PSA) detection of $>0.1 \mathrm{ng} /$ $\mathrm{ml}$ in at least two consecutive tests. DFS was defined as the length of time between the date of prostatectomy to the date of death or last follow-up.

\section{Genotyping of Aurora Kinase A polymorphisms in prostate cancer samples}

Prostatectomy specimens were processed and genomic DNA extracted from deparaffinizedspecimens as described previously [15]. Purified genomic DNA was genotyped for the following haplotypetagging SNPs in the AURKA gene: $r s 1468055, r s 8117896, r s 2064863$, and $r s 1468056$. Haplotype-tagging SNPs (htSNPs) were selected using the ABI SNP Browser to cover SNPs with an $\mathrm{r}^{2}$ of 0.90 or greater in the AURKA gene with a minor allele frequency (MAF) of at least 0.25. A total of 4 SNPs were selected and genotyped in our patient cohort. Allelic discrimination of these AURKA polymorphisms was performed using Taqman SNP genotyping assays (Applied Biosystems: C_31520284_10 [rs8117896], C_26713921_10 [rs2064863], and C_8947664_10 [rs1468056]). The final volume for each reaction was $5 \mu \mathrm{l}$, consisting of $2.5 \mu \mathrm{l}$ TaqMan Universal PCR Master Mix (Applied Biosystems), $900 \mathrm{nM}$ of each primer, $200 \mathrm{nM}$ of each TaqMan probe, and 5ng genomic DNA. The PCR profile consisted of an initial denaturation step at $95^{\circ} \mathrm{C}$ for $10 \mathrm{~min}$ and 50 cycles with $95^{\circ} \mathrm{C}$ for 15 sec and $60^{\circ} \mathrm{C}$ for $1 \mathrm{~min}$. The fluorescence level was measured with the ABI PRISM 7900HT sequence detector. Genotypes were determined utilizing ABI SDS software. Quality control samples were included in genotyping assays.

\section{Statistical analysis}

Statistical analyses were executed to detect associations between the four AURKA htSNPs and RFS/DFS. SNPs associated with survival outcomes were evaluated based on log-rank tests. DFS was defined as the length of time between the date of prostatectomy to the date of death or last follow-up, where death events were defined as patients who died of prostate cancer only. RFS was defined as the interval between the date of surgery and the date of recurrence or last follow-up. Data were censored for live (or recurrence-free) patients as of their last follow-up visits. Kaplan-Meier survival curves were calculated for the subgroups of potential risk factors and were compared using the log-rank test. For multivariable analysis, Cox proportional hazards model was applied to investigate the association between SNPs and RFS with adjusting for PSA, surgical margin, and grade. Descriptive statistics, including mean and standard deviation for continuous variables, as well as percentages and frequencies for categorical variables, were calculated (Table 1). All $P$ values are based on two-sided tests, and differences were considered statistically significant when $p$ value $<0.05$. Analyses were performed using SAS system version 9.1 and $\mathrm{R}$ version 2.8.

\section{Bioinformatics tools}

Web-based databases used in the present study include NCBI Database of Single Nucleotide Polymorphisms (dbSNP) [Build 129, http://www.ncbi.nlm.nih.gov/SNP] and the International HapMap Project [16], for its allele frequency data in the CEU population (i.e., Utah residents with ancestry from Northern and Western Europe) and for its genome browser and Haploview software to measure the extent of linkage disequilibrium (LD) between SNPs and generate an LOD

\begin{tabular}{|c|c|}
\hline Characteristic & Number $(\mathrm{N}=\mathbf{2 1 2})$ \\
\hline \multicolumn{2}{|l|}{ Race, $n(\%)$} \\
\hline White & $208(98.11)$ \\
\hline Black & $4(1.89)$ \\
\hline \multicolumn{2}{|l|}{ Age at diagnosis, $\mathrm{n}(\%)$, years } \\
\hline Mean (SD) & $61.2(7.08)$ \\
\hline$<60$ & $86(40.57)$ \\
\hline $60-70$ & $104(49.06)$ \\
\hline$>70$ & $22(10.38)$ \\
\hline \multicolumn{2}{|l|}{ Pre-prostatectomy PSA, n(\%), ng/ml } \\
\hline Mean (SD) & $8.4(6.21)$ \\
\hline$<4$ & $22(10.38)$ \\
\hline $4-10$ & $113(53.30)$ \\
\hline$>10$ & $43(20.28)$ \\
\hline Missing & $34(16.04)$ \\
\hline \multicolumn{2}{|l|}{ Gleason score, n(\%) } \\
\hline $2-6$ & $130(61.32)$ \\
\hline 7 & $70(33.02)$ \\
\hline $8-10$ & $12(5.66)$ \\
\hline \multicolumn{2}{|l|}{ Surgical margin, n(\%) } \\
\hline Negative & $133(62.74)$ \\
\hline Positive & $78(36.79)$ \\
\hline Missing & $1(0.47)$ \\
\hline \multicolumn{2}{|l|}{ Extracapsular extension, n(\%) } \\
\hline Negative & $125(58.96)$ \\
\hline Positive & $67(31.60)$ \\
\hline Missing & $20(9.43)$ \\
\hline \multicolumn{2}{|l|}{ Disease classification ${ }^{1}, \mathrm{n}(\%)$} \\
\hline Clinically inapparent (T1) & $8(3.77)$ \\
\hline Confined within prostate (T2) & $134(63.21)$ \\
\hline Regional (T3) & $5(26.89)$ \\
\hline Missing & $13(6.13)$ \\
\hline \multicolumn{2}{|l|}{ Recurrence, $\mathrm{n}(\%)$} \\
\hline No & $168(79.25)$ \\
\hline Yes & $44(20.75)$ \\
\hline \multicolumn{2}{|l|}{ Disease-free survival, $n(\%)$} \\
\hline No & $5(2.36)$ \\
\hline Yes & $207(97.64)$ \\
\hline
\end{tabular}

Abbreviations: PSA: Prostate-specific Antigen

${ }^{1}$ Based on the American Joint Committee on Cancer classification.

Table 1: Patient Demographics. 
Citation: Jaboin JJ, Ausborn NL, Hwang M, Chen H, Niermann KJ, et al. (2012) The Aurora Kinase A Polymorphisms are not Associated with Recurrence-free Survival in Prostate Cancer Patients. J Cancer Sci Ther 4: 016-022. doi:10.4172/1948-5956.1000105

score plot [http://www.hapmap.org]. The HapMap datasource used was HapMap Data Rel 23a/Phase II Mar 08, on NCBI B36 Assembly, dbSNPb126.

\section{Results}

\section{Patient characteristics}

Demographic, clinicopathologic, and outcomes information are summarized in Table 1. The mean age of study subjects at the time of prostate cancer diagnosis and definitive treatment with prostatectomy was $61.2 \pm 7-y$. After a median follow-up time of $9.1-y, 83 \%$ of the patients were alive. The median follow-up time for prostate cancer progression was $3.4-y$. The recurrence rate was $21 \%$, with the majority classified as biochemical and a lower incidence for local or distant recurrence ( $80 \%, 5 \%$ and $16 \%$ of recurrences, respectively). Based on the risk stratification scheme suggested by the National Comprehensive Cancer Network ${ }^{\circledast}$ guidelines [17], which incorporates Gleason grade, PSA levels and staging parameters to predict probability of biochemical failure after definitive local therapy, the majority of our patient population (64\%) could be classified as having an intermediate risk for biochemical failure.

Lack of association between intronic AURKA SNPs and recurrence and survival outcomes in prostate cancer

Four intronic AURKA SNPs $r s 1468055, r s 8117896, r s 2064863$, and rs1468056 were genotyped to examine their association with prognostic outcomes in prostate cancer treated with radical prostatectomy (Table 2). For the majority of SNPs, allelic discrimination assays were informative for at least $86 \%$ of genomic DNA samples. The genotype frequency distribution for the SNPs in our study population closely matched the one previously reported in the HapMap database for the CEU population [16], adding confidence to the accuracy of our genotyping reaction.

A log-rank univariate analysis was performed to identify significant associations between RFS or DFS and known prognostic factors as well as AURKA genotypes, which is shown in Table 3. Prognostic factors analyzed, including pre-prostatectomy PSA levels $(\mathrm{p}<0.001)$, Gleason score $(p<0.001)$, surgical margin $(\mathrm{p}<0.001)$, and AJCC tumor category $(p<0.001)$ [17] showed statistically significant associations with RFS.
In the present study, only Gleason score was significantly associated with differential probability of DFS $(p<0.001)$. No statistically significant associations were found for the SNPs $r s 1468055, r s 8117896$, $r s 2064863$, and $r s 1468056$ analyzed for either RFS $(\mathrm{p}=0.7213, \mathrm{p}=0.5140$, $\mathrm{p}=0.0714, \mathrm{p}=0.4731$, respectively) or DFS $(\mathrm{p}=0.3282, \mathrm{p}=0.1909$, $\mathrm{p}=0.4111, \mathrm{p}=0.5014$, respectively). The Kaplan-Meier method was used to estimate the probability distribution of RFS as a function of genotype for each SNP, and the log-rank test was used to determine the significance of RFS differences across the different genotypes (Figure 1). No statistically significant genotypes were demonstrated.

Additionally, a Cox proportional hazards model was used to identify associations between AURKA SNPS and RFS. Due to a limitation of only 5 events for DFS, a multivariable model could not be obtained for DFS. Hazards ratios for SNPs $r s 1468055, r s 8117896, r s 2064863$, and rs1468056 ranged from 0.489 to 1.004 and are shown in Table 4.

\section{Discussion}

Aurora Kinase A (AURKA) is a serine/threonine kinase that functions to ensure a proper G2-M transition in cell cycle. AURKA has been accepted as an oncogene [reviewed in 18], with its overexpression leading to centrosomal and chromosomal instability with subsequent accretion of invasive and malignant phenotypes in many human cancer types $[1,2]$. Some studies have associated AURKA overexpression with early prostate tumorigenesis due to a higher AURKA expression level in high-grade prostate intraepithelial neoplasia (PIN) than in advanced tumors [19]. In other studies, upregulation of AURKA has shown to promote prostate cancer metastasis [20]. At present, the mechanistic details on how AURKA overexpression contributes to cancer progression remains largely unknown.

Over the years, the list of biomarkers with potential prognostic implication in prostate cancer has grown [21]. With AURKA in particular, several single-nucleotide polymorphisms (SNPs) have been identified to contribute to adverse outcomes in cancer. One most studied functional AURKA SNP is $r 22273535$ resulting in a T91A change at codon 31 and displaying an independent risk-enhancing effect in multiple tumors including colon, breast, prostate, skin, lung and esophagus [22]. Another functional AURKA SNP is rs 1047972 with

\begin{tabular}{|c|c|c|c|c|c|c|c|c|c|c|c|}
\hline \multirow{4}{*}{$\begin{array}{l}\text { dbSNP ID }^{1} \\
r s 1468055\end{array}$} & \multirow{4}{*}{\begin{tabular}{|c|} 
Chromosomal Position \\
Chr20:54,399,308 \\
\end{tabular}} & \multirow{4}{*}{$\begin{array}{c}\text { Location } \\
\text { (relative to } \\
\text { AURKA) }^{3}\end{array}$} & \multirow{4}{*}{$\begin{array}{c}\text { Alleles }^{1} \\
\text { C/A }\end{array}$} & \multirow{4}{*}{\begin{tabular}{|c|}
$\begin{array}{c}\text { Amino Acid } \\
\text { Change }\end{array}$ \\
N/A \\
\end{tabular}} & \multicolumn{7}{|c|}{ Population Diversity } \\
\hline & & & & & \multicolumn{4}{|c|}{ Genotype Frequency in Study Population } & \multirow{2}{*}{\multicolumn{3}{|c|}{$\begin{array}{c}\text { HapMap-CEU Genotype } \\
\text { Frequency }^{1} \\
\text { Genotypes }^{\text {Gety }}\end{array}$}} \\
\hline & & & & & \multirow{2}{*}{$\begin{array}{c}\text { Patient No. }^{4} \\
194\end{array}$} & \multicolumn{3}{|c|}{ Genotypes } & & & \\
\hline & & & & & & $\begin{array}{c}C / C \\
0.585\end{array}$ & $\begin{array}{c}C / A \\
0.250\end{array}$ & $\begin{array}{c}A / A \\
0.080\end{array}$ & $\begin{array}{c}C / C \\
0.567\end{array}$ & $\begin{array}{c}C / A \\
0.367\end{array}$ & $\begin{array}{c}A / A \\
0.067\end{array}$ \\
\hline rs1468056 & Chr20:54,399,395 & Intron & $C / G$ & $\mathrm{~N} / \mathrm{A}$ & 156 & $\begin{array}{c}C / C \\
0.137\end{array}$ & $\begin{array}{c}C / G \\
0.349\end{array}$ & $\begin{array}{c}G / G \\
0.349\end{array}$ & $\begin{array}{c}C / C \\
0.133\end{array}$ & $\begin{array}{c}C / G \\
0.533\end{array}$ & $\begin{array}{c}G / G \\
0.333\end{array}$ \\
\hline$r s 8117896$ & Chr20:54,389,471 & Intron & $\mathrm{C} / \mathrm{T}$ & N/A & 146 & $\begin{array}{c}C / C \\
0.330\end{array}$ & $\begin{array}{c}C / C \\
0.198\end{array}$ & $\begin{array}{c}T / T \\
0.160\end{array}$ & $\begin{array}{c}C / C \\
0.417\end{array}$ & $\begin{array}{c}C / T \\
0.433\end{array}$ & $\begin{array}{c}T / T \\
0.150\end{array}$ \\
\hline rs2064863 & Chr20:54,396,179 & Intron & $A / C$ & $\mathrm{~N} / \mathrm{A}$ & 200 & $\begin{array}{c}A / A \\
0.202\end{array}$ & $\begin{array}{c}A / C \\
0.392\end{array}$ & $\begin{array}{c}C / C \\
0.349\end{array}$ & $\begin{array}{c}A / A \\
0.150\end{array}$ & $\begin{array}{c}A / C \\
0.500\end{array}$ & $\begin{array}{c}C / C \\
0.350\end{array}$ \\
\hline
\end{tabular}

Abbreviations: AURKA: Aurora Kinase A; dbSNP: Database Single Nucleotide Polymorphism; chr: Chromosome; HapMap: International HapMap Project; CEU: Population Consisting of Utah Residents with Ancestry from Northern and Western Europe

1Data obtained from NCBI Database of Single Nucleotide Polymorphisms (dbSNP) Build ID: 129, http://www.ncbi.nlm.nih.gov/SNP/, accessed December 2008. Ancestral allele is written first.

${ }^{2}$ Based on alignment with the NCBI Build 36.1 human reference sequence using the BLAT software, http://genome.ucsc.edu/, accessed December 2008.

${ }^{3}$ Relative to annotated genomic sequence for human AURKA, chr20: 20q13.2-q13.3.

${ }^{4}$ Indicates number of patients $(\mathrm{n}=212)$ for which the genotyping sequence yielded informative results.

Table 2: AURKA SNP Frequencies among Prostate Cancer Patients. 
Citation: Jaboin JJ, Ausborn NL, Hwang M, Chen H, Niermann KJ, et al. (2012) The Aurora Kinase A Polymorphisms are not Associated with Recurrence-free Survival in Prostate Cancer Patients. J Cancer Sci Ther 4: 016-022. doi:10.4172/1948-5956.1000105

\begin{tabular}{|c|c|c|c|c|c|}
\hline \multirow{2}{*}{ Factor } & \multirow{2}{*}{$\begin{array}{c}\text { Number of } \\
\text { Patients }^{1}(n=212)\end{array}$} & \multicolumn{2}{|c|}{ 3-Yrs Recurrence-Free } & \multicolumn{2}{|c|}{ 5-Yrs Disease-Free Survival } \\
\hline & & $\%(95 \% \mathrm{Cl})$ & P Value $^{2}$ & $\%(95 \% \mathrm{Cl})$ & P Value \\
\hline Race & & & 0.8354 & & 0.7082 \\
\hline Black & 4 & $N A^{3}$ & & $N A^{3}$ & \\
\hline White & 208 & $88(83-92)$ & & $98(96-100)$ & \\
\hline Age at diagnosis (years) & & & 0.8123 & & 0.1935 \\
\hline $60-70$ & 104 & $85(78-92)$ & & $N A^{3}$ & \\
\hline$<=60$ & 86 & $89(83-96)$ & & $97(93-100)$ & \\
\hline$>70$ & 22 & $81(65-98)$ & & $N A^{3}$ & \\
\hline Pre-prostatectomy PSA (ng/ml) & & & 0.0434 & & 0.6713 \\
\hline $4-10$ & 113 & $88(82-94)$ & & $97(93-100)$ & \\
\hline$<4$ & 22 & $N A^{3}$ & & $\mathrm{NA}^{3}$ & \\
\hline$>10$ & 43 & $84(72-95)$ & & $96(87-100)$ & \\
\hline Gleason score & & & $<0.001$ & & $<0.001$ \\
\hline $2-6$ & 130 & $94(89-98)$ & & $N A^{3}$ & \\
\hline 7 & 70 & $77(67-87)$ & & $98(93-100)$ & \\
\hline $8-10$ & 12 & $67(40-93)$ & & $71(43-100)$ & \\
\hline Surgical margin & & & $<0.001$ & & 0.3690 \\
\hline Negative & 133 & $95(91-98)$ & & $99(96-100)$ & \\
\hline Positive & 78 & $74(64-84)$ & & $95(90-100)$ & \\
\hline Extracapsular extension & & & $<0.001$ & & 0.2664 \\
\hline Negative & 125 & $93(88-97)$ & & $99(96-100)$ & \\
\hline Positive & 67 & $76(65-86)$ & & $94(88-100)$ & \\
\hline Disease Stage & & & $<0.001$ & & 0.1248 \\
\hline Localized (T1) & 8 & $71(38-100)$ & & $N A^{3}$ & \\
\hline Localized (T2) & 134 & $94(90-98)$ & & $99(96-100)$ & \\
\hline Localized (T3) & 57 & $71(59-83)$ & & $95(89-100)$ & \\
\hline AURKA SNP rs 1468055 & & & 0.7213 & & 0.3282 \\
\hline $\mathrm{A} / \mathrm{A}$ & 17 & $76(57-100)$ & & $N A^{3}$ & \\
\hline $\mathrm{A} / \mathrm{C}$ & 53 & $84(74-94)$ & & $97(91-100)$ & \\
\hline $\mathrm{C} / \mathrm{C}$ & 124 & $88(83-94)$ & & $98(95-100)$ & \\
\hline AURKA SNP rs8117896 & & & 0.5140 & & 0.1909 \\
\hline $\mathrm{C} / \mathrm{C}$ & 70 & 87 (79- 85) & & $96(91-100)$ & \\
\hline $\mathrm{C} / \mathrm{T}$ & 42 & $82(70-94)$ & & $N A^{3}$ & \\
\hline $\mathrm{T} / \mathrm{T}$ & 34 & 88 (77- 99) & & $N A^{3}$ & \\
\hline AURKA SNP rs2064863 & & & 0.0714 & & 0.4111 \\
\hline $\mathrm{G} / \mathrm{G}$ & 74 & $83(75-92)$ & & $97(92-100)$ & \\
\hline $\mathrm{G} / \mathrm{T}$ & 83 & $89(82-96)$ & & $97(92-100)$ & \\
\hline $\mathrm{T} / \mathrm{T}$ & 43 & $88(78-98)$ & & $\mathrm{NA}^{3}$ & \\
\hline AURKA SNP rs 1468056 & & & 0.4731 & & 0.5014 \\
\hline $\mathrm{C} / \mathrm{C}$ & 29 & $73(55-90)$ & & $N A^{3}$ & \\
\hline $\mathrm{C} / \mathrm{G}$ & 53 & $89(80-97)$ & & $97(92-100)$ & \\
\hline $\mathrm{G} / \mathrm{G}$ & 74 & $90(83-97)$ & & $\mathrm{NA}^{3}$ & \\
\hline
\end{tabular}

${ }^{1}$ Analysis were limited to patients for whom data were available.

${ }^{2} \mathrm{P}$ values for each factor were calculated by the log-rank test.

${ }^{3}$ Unable to calculate because all recurrence or death events occurred before 3-y or 5-y, respectively.

Table 3: Effect of Patient Factors on Post-prostatectomy Outcomes, Univariate Analysis. 

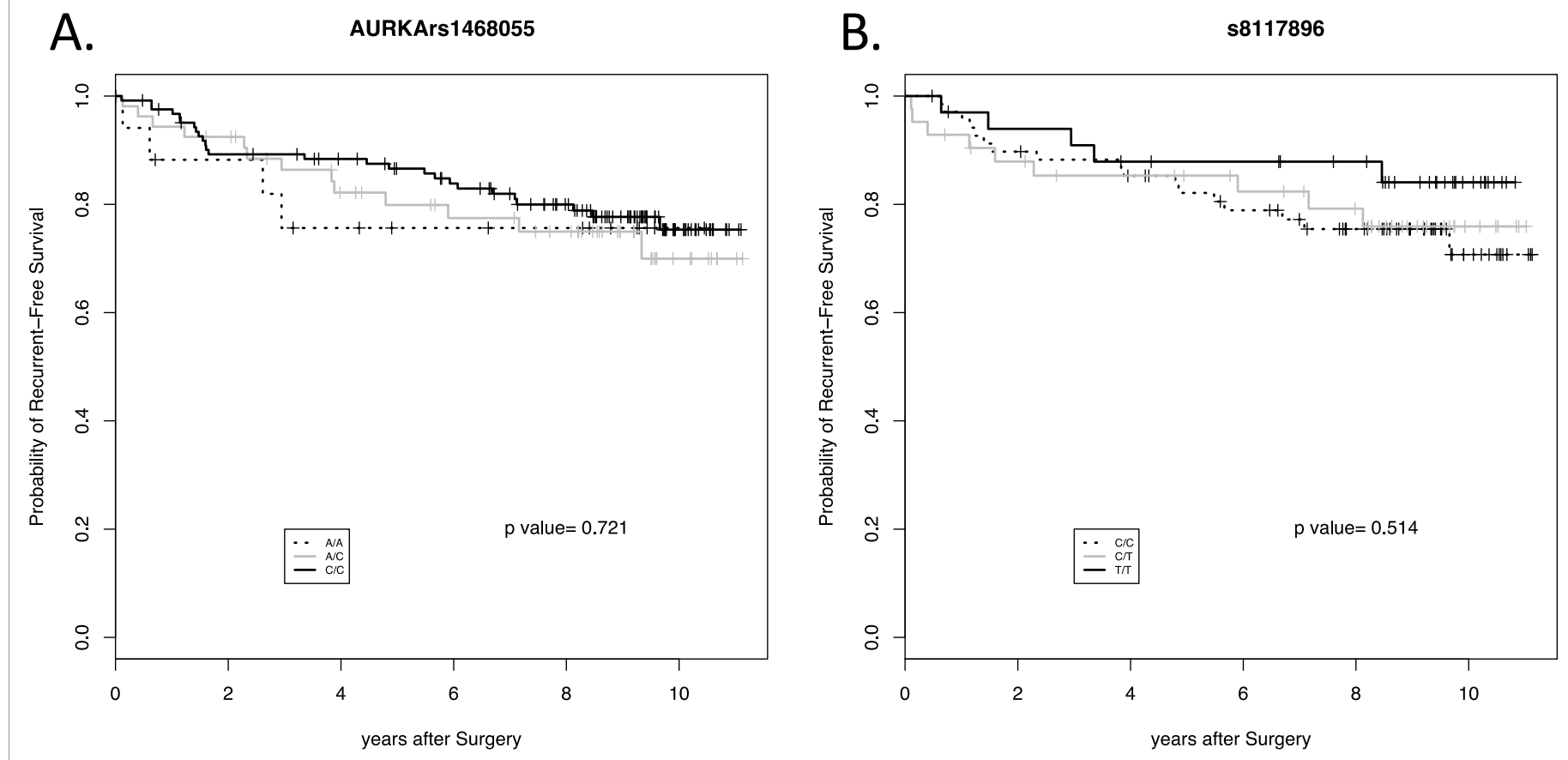

C.

AURKArs2064863
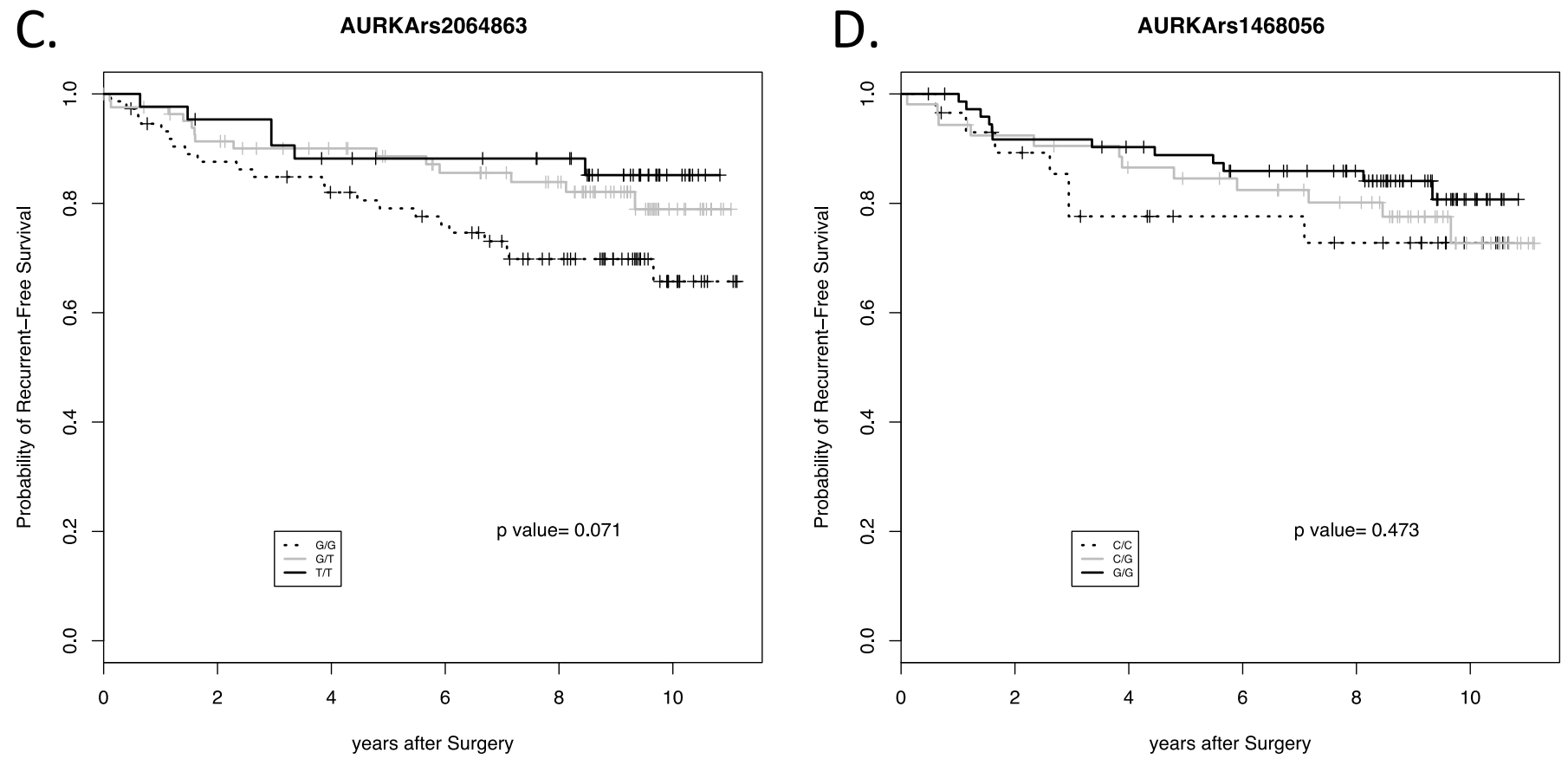

Figure 1: Kaplan-Meier estimates of recurrence-free survival following radical prostatectomy treatment for localized prostate cancer. Recurrence-free survival curves were plotted for the individual genotypes for each AURKA SNP: (A) SNP rs 1468055 (B) SNP rs8117896 (C) SNP rs2064863 (D) SNP rs1468056.

a $A 169 G$ change shown to occur more frequently in advanced than in early gastric cancers [9]. The same study also noted that its effects on gastric cancer progression are related to the enhancement of relative kinase activities of AURKA variants. Such SNP studies will not only provide the starting point for unraveling the molecular mechanisms behind tumor aggression, but also equip us with accurate biomarkers for patient-tailored risk assessment and treatment.

This retrospective study demonstrated no evidence for intronic AURKA SNPs $r s 1468055, r s 8117896$, rs 2064863 , or $r s 1468056$ in predicting recurrence-free survival or disease-free survival in patients with prostate cancer. There are several limitations in our study. First, tumor registry data examining the causes of death yielded 
Citation: Jaboin JJ, Ausborn NL, Hwang M, Chen H, Niermann KJ, et al. (2012) The Aurora Kinase A Polymorphisms are not Associated with Recurrence-free Survival in Prostate Cancer Patients. J Cancer Sci Ther 4: 016-022. doi:10.4172/1948-5956.1000105

\begin{tabular}{|l|l|l|l|}
\hline & P Value & \multicolumn{2}{|c|}{ Hazards Ratio (95\% CI) $^{\mathbf{1}}$} \\
\hline AURKA SNP rs1468055 & 0.7018 & A/C & $0.613(0.189-1.991)$ \\
\hline \multirow{2}{*}{ AURKA SNP rs8117896 } & \multirow{2}{*}{0.9786} & C/C & $0.657(0.225-1.921)$ \\
\hline AURKA SNP rs2064863 & C/T & $1.004(0.398-2.530)$ \\
\hline AURKA SNP rs1468056 & \multirow{2}{*}{0.1585} & T/T & $0.902(0.319-2.551)$ \\
\hline
\end{tabular}

${ }^{1} \mathrm{P}$ values were calculated by Chi-square anaylysis.

${ }^{2}$ Hazards ratio calculated by Cox proportional hazards model. $\mathrm{Cl}=$ confidence interval.

Table 4: Recurrence-Free Survival, Multivariable Analysis.

only 5 confirmed prostate cancer deaths out of the 40 reported deaths in the reviewed patient population. This could especially have an impact on calculation of disease-free survival. In addition, 20 patients had unknown causes of death. A multivariate analysis of only the known deaths with independent prognosticators of prostate cancer did not achieve statistical significance (data not shown). Various confounders contributing to the death of prostate cancer patients need to be taken into account before any statement about the SNP significance on survival outcome is made. Secondly, the population size may have limited the power to detect existing associations between the SNPs studied and the prostate cancer outcomes, contributing to a possible false negative result. The study population was also primarily Caucasian (98\%) which may limit the application of this genetic marker across different races. Future validation studies are warranted.

Despite these limitations, our study examined the potential clinical significance of intronic AURKA SNPs in prostate cancer outcome for the first time. Additional patient numbers and longer follow-up may allow detection of a statistical difference in RFS or DFS with AURKA SNP genotype in the event that our results yielded a false negative due to these limitations. In addition, future studies with a complete compilation of the causes of death will allow a more accurate assessment of the role of these SNPs in prostate cancer progression and outcome.

\section{Conclusions}

Our study examined the potential clinical significance of intronic AURKA SNPs in prostate cancer outcome for the first time. These findings have implications to the state of the field with the demonstration for the lack of association between intronic AURKA SNPs as biomarkers for predicting recurrence and survival outcomes in patients with prostate cancer.

\section{Authors' Contributions}

JJJ extracted the DNA, participated in the updating of the database, participated in the design of the study, and generated the list of haplotypetagging SNPs. NLA assisted in interpretation of the data analysis and writing of the manuscript. $\mathrm{MH}$ assisted with stastistcal analysis and writing the intital draft of the paper. HC provided statistical analysis. KJN participated in the design of the study and its coordination and helped draft the manuscript. NJG assisted in the analysis of the data and preparation of the manuscript. RC and QC carried out the genotyping. BCT revised the manuscript. BL participated in study design, discussed analyses, interpretation, and presentation. All authors read and approved the final manuscript.

\section{Acknowledgements}

We thank Dr. Marcia Wills and Judith N. Roberts for their assistance with collection of specimens and clinical survival data, respectively. This work was supported by CA093240-06 and VICTR CTSA (Grant\# 1KL2RR024977-01, Voucher: 194).

\section{References}

1. Sen S, Zhou H, Zhang RD, Yoon DS, Vakar-Lopez F, et al. (2002) Amplification/ overexpression of a mitotic kinase gene in human bladder cancer. $\mathrm{J}$ Nat Cancer Inst 94: 1320-1329.

2. Tanaka T, Kimura M, Matsunaga K, Fukada D, Mori H, et al. (1999) Centrosomal kinase AlK1 is overexpressed in invasive ductal carcinoma of the breast. Cancer Res 59: 2041-2044.

3. Zhou H, Kuang J, Zhong L, Kuo WL, Gray JW, et al. (1998) Tumour amplified kinase STK15/BTAK induces centrosome amplification, aneuploidy and transformation. Nat Genet 20: 189-193.

4. Lee EC, Frolov A, Li R, Ayala G, Greenberg NM (2006) Targeting Aurora kinases for the treatment of prostate cancer. Cancer Res 66: 4996-5002.

5. Matarasso N, Bar-Shira A, Rozovski U, Rosner S, Orr-Urtreger A (2007) Functional analysis of the Aurora Kinase A lle31 allelic variant in human prostate. Neoplasia 9: 707-715.

6. Hoque A, Carter J, Xia W, Hung MC, Sahin AA, et al. (2003) Loss of aurora A/STK15/BTAK overexpression correlates with transition of in situ to invasive ductal carcinoma of the breast. Cancer Epidemiol Biomarkers Prev 12: 15181522.

7. Royce ME, Xia W, Sahin AA, Katayama H, Johnston DA, et al. (2004) STK15/ Aurora-A expression in primary breast tumors is correlated with nuclear grade but not with prognosis. Cancer 100: 12-19.

8. Li D, Zhu J, Firozi PF, Abbruzzese JL, Evans DB, et al. (2003) Overexpression of oncogenic STK15/BTAK/Aurora A kinase in human pancreatic cancer. Clin Cancer Res 9: 991-997.

9. Ju H, Cho H, Kim YS, Kim WH, Ihm C, et al. (2006) Functional polymorphism $57 \mathrm{Val}>\mathrm{lle}$ of aurora kinase A associated with increased risk of gastric cancer progression. Cancer Lett 242: 273-279.

10. Miao X, Sun T, Wang Y, Zhang X, Tan W, et al. (2004) Functional STK15 Phe31lle polymorphism is associated with the occurrence and advanced disease status of esophageal squamous cell carcinoma. Cancer Res 64: 26802683.

11. Gu J, Gong Y, Huang M, Lu C, Spitz MR, et al. (2007) Polymorphisms of STK15 (Aurora-A) gene and lung cancer risk in Caucasians. Carcinogenesis 28: 350 355.

12. Peters CA, Stock RG, Cesaretti JA, Atencio DP, Peters S, et al. (2008) TGFB1 single nucleotide polymorphisms are associated with adverse quality of life in prostate cancer patients treated with radiotherapy. Int J Radiat Oncol Biol Phys 70: 752-759.

13. Donovan MJ, Hamann S, Clayton M, Khan FM, Sapir M, et al. (2008) Systems pathology approach for the prediction of prostate cancer progression after radical prostatectomy. J Clin Oncol 26: 3923-3929.

14. Jhaveri FM, Zippe CD, Klein EA, Kupelian PA (1999) Biochemical failure does not predict overall survival after radical prostatectomy for localized prostate cancer: 10-year results. Urology 54: 884-890.

15. Browning RE 4th, Li H, Shinohara ET, Cai Q, Chen H, et al. (2006) ATM 
Citation: Jaboin JJ, Ausborn NL, Hwang M, Chen H, Niermann KJ, et al. (2012) The Aurora Kinase A Polymorphisms are not Associated with Recurrence-free Survival in Prostate Cancer Patients. J Cancer Sci Ther 4: 016-022. doi:10.4172/1948-5956.1000105

polymorphism IVS62+60G $>A$ is not associated with disease aggressiveness in prostate cancer. Urology 67: 1320-1323.

16. The International HapMap Consortium (2003) The International HapMap Project. Nature 426: 789-796.

17. Greene FL, Page DL, Fleming ID, Fritz AG, Balch CM, et al. (Eds) (2002) AJCC Cancer Staging Manual. (6thedn), Springer, New York.

18. Carmena M, Earnshaw WC (2003) The cellular geography of aurora kinases. Nat Rev Mol Cell Biol 4: 842-854.

19. Buschhorn HM, Klein RR, Chambers SM, Hardy MC, Green S, et al. (2005) Aurora-A over-expression in high-grade PIN lesions and prostate cancer. Prostate 64: 341-346.
20. Bar-Shira A, Pinthus JH, Rozovsky U, Goldstein M, Sellers WR, et al. (2002) Multiple genes in human $20 q 13$ chromosomal region are involved in an advanced prostate cancer xenograft. Cancer Res 62: 6803-6807.

21. D’Amico F, Biancolella M, Margiotti K, Reichardt JK, Novelli G (2007 Genomic biomarkers, androgen pathway and prostate cancer. Pharmacogenomics 8 : 645-661.

22. Ewart-Toland A, Dai Q, Gao YT, Nagase H, Dunlop MG, et al. (2005) Aurora-A STK15 T+91A is a general low penetrance cancer susceptibility gene: a metaanalysis of multiple cancer types. Carcinogenesis 26: 1368-1373. 